\title{
MATHEMATICAL MODELLING OF STATIONARY THERMOELASTIC STATE FOR A PLATE WITH PERIODIC SYSTEM OF INCLUSIONS AND CRACKS
}

\author{
Volodymyr ZELENYAK \\ *Department of Mathematics, Institute of Applied Mathematics and Fundamental Sciences, Lviv Polytechnic National University, \\ S. Bandery str., 12, 79013, Lviv, Ukraine \\ volodymyr.zelenyak@gmail.com
}

received 8 November 2018, revised 3 March 2019, accepted 7 March 2019

\begin{abstract}
Two-dimensional stationary problem of heat conduction and thermoelasticity for infinite elastic body containing periodic system of inclusions and cracks is considered. Solution of the problem is constructed using the method of singular integral equations (SIEs). The numerical solution of the system integral equations are obtained by the method of mechanical quadrature for a plate heated by a heat flow, containing periodic system elliptic inclusions and thermally insulated cracks. There are obtained graphic dependences of stress intensity factors (SIFs), which characterise the distribution of intensity of stresses at the tops of a crack, depending on the length of crack, elastic and thermoelastic characteristics inclusion, relative position of crack and inclusion.
\end{abstract}

Keywords: Stress Intensity Factor, Singular Integral Equation, Inclusion, Heat Conduction, Thermoelasticity, Crack

\section{INTRODUCTION}

Singular integral equations (SIEs) are often applied in studying the stressed state of bodies with cracks. First, they were used only for very simple rectilinear and arcwise cuts. The construction of equations of this kind for systems of rectilinear cracks arbitrarily located in the elastic plane became a substantial step forward in this direction. The results obtained enabled the researchers to find the numerical and analytic solutions of the plane and antiplane problems of the theory of elasticity, the steady-state problems of heat conduction and thermoelasticity and the problems of bending of thin plates and gently sloping shells for domains with cuts (Panasyuk et al., 1976). Later, these results were generalised to the case of curvilinear cuts (cracks) and multiply connected domains with holes and cuts of any configuration (Savruk,1981).

However, for homogeneous bodies with cracks (cuts), the problems of thermoelasticity remain poorly investigated, and, in particular, in the available literature, one can find almost no solution for noncircular (e.g. elliptic) inclusions and especially for multicomponent bodies with cracks.

The two-dimensional problems of thermoelasticity for semiinfinite bodies with cracks have already been investigated in the literature. Thus, in particular, the thermoelastic state of a half plane containing an internal rectilinear crack was investigated, and different temperature and force conditions imposed on the crack lips and on the edge of the half plane were analysed in (Sekine, 1975; Tweed and Love, 1979). The method of SIE was used for the analysis of the plane thermoelastic state in a half space locally heated over a part of its free surface by a heat flow and containing an internal arbitrary-oriented rectilinear crack or a periodic system of cracks of this sort (Matysiak et al,1999) or internal curvilinear crack (Zelenyak and Kolyasa, 2016) .

The SIE for stationary problems of heat conduction and thermoelasticity for infinite and finite (Savruk and Zelenyak,1987) piecewise homogeneous planes weakened by curvilinear cracks with given temperature or heat flows specified on their lips are deduced by the method of integral representations of complex temperature and stress potentials. The SIE of heat conduction and thermoelasticity with special Cauchy-type kernels for a plane with thermally insulated cracks or heat-conducting cuts located in a circular foreign inclusion; for a body with thermal cylindrical inclusions and crack (Kit and Chernyak,2010); for an infinite body with two identical coplanar thermally active circular cracks (Sushko, 2013); and for a half space locally heated over a part of its free surface by a heat flow and containing an internal rectilinear crack (Kit and lvas'ko, 2013) are deduced by the method of functions of complex variable. The mechanical problem of interaction of a curvilinear crack with a circular inclusion in an infinite elastic plane is studied in Cheesman and Santare (2000). The distribution of temperature stresses near the crack tips in threecomponent region (i.e. in a plane containing a foreign twocomponent inclusion and crack) at a constant temperature was studied in Zelenyak (2012). The two-dimensional elastic problem in a similar three-component region was presented in (Xiao and Chen, 2001). The method of SIEs was used for finding the solution of two-dimensional problems of thermoelasticity for a threelayer circular hollow cylinder weakened by a crack (Zelenyak, 2015). The solutions of the thermoelasticity problem for a plane with a crack based on the edge method (Chen et al., 2016) and the Fourier integral transform method (Choi et al., 2014) was presented.

Dynamic steady-state growth in 3D of a semi-infinite plane brittle crack in a coupled thermoelastic solid is considered. An asymptotic solution is obtained in an analytic form and subjected to a criterion of the Griffith type for the case of a compressive point force (Brock, 2016). The multiple cracks problem in an elastic half-plane is formulated into SIE using the modified complex potential with free traction boundary condition. A system of SIEs is 
obtained with the distribution dislocation function as unknown and the traction applied on the crack faces as the right-hand terms (Elfakhakhre, 2017). The problem of the stress concentration in the vicinity crack tips for a crack of finite length located perpendicular to the interface of two elastic bodies - a half-plane and a strip - is considered. Using the method of generalised integral transforms, the problem reduces to the solution of an SIE with a Cauchy kernel (Rashidova and Sobol, 2017). Local frictional heating of surface of a layered plate with inclusion was investigated in Havrysh $(2017,2015)$.

The aim of this article is (1) to construct a two-dimensional mathematical model of the stationary problems of heat conduction and thermoelasticity for infinite elastic body containing periodic system inclusions and cracks in the form of system SIE; (2) to determine the numerical solutions to SIE (using the method of mechanical quadratures) in particular case of periodic system elliptic inclusions and thermally insulated cracks. This makes it possible to determine stress intensity factors (SIFs) at the tops of the crack, which are subsequently used to determine critical values of the heat flow at which a crack starts to grow.

\section{PROBLEM STATEMENT}

Consider an infinite body consisting of a matrix $S$ and a periodic system of inclusions and cracks, and in the main band of periods of width $\mathrm{d}$ (along the axis $O x$ ), there are $\mathrm{M}$ inclusions, bounded by smooth closed contours $L_{k}(k=\overline{1, M})$ and N-M curvilinear cracks $L_{k}(k=\overline{M+1, N})$, located in both the matrix and the inclusions. We assume that all contours $L_{k}(k=\overline{1, N})$ do not have common points. Each contour is connected with a local coordinate system $x_{k} O_{k} y_{k}$ whose axis $O_{k} x_{k}$ forms an angle $\alpha_{k}$ with the axis $O x$, and the points $O_{k}$ are determined in the coordinate system xOy by complex coordinates $z_{k}^{0}$. Bypassing the contours $L_{k}(k=\overline{1, M})$ in a positive direction, the area $S_{k}$ remains to the left (Fig. 1).

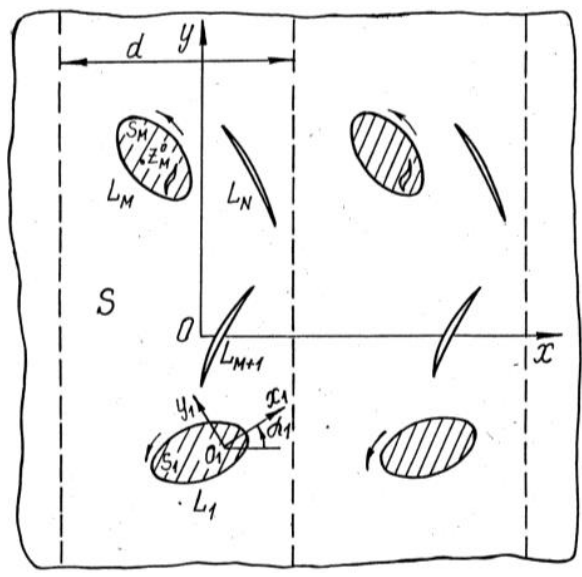

Fig. 1. Geometric scheme of the problem

Consider the problem of heat conduction with the following conditions of thermal contact. We will assume that along the closed contours, the conditions for an ideal thermal contact (temperature and heat equivalence) are given:

$\lambda_{k} \frac{\partial T^{+}}{\partial n}=\lambda \frac{\partial T^{-}}{\partial n}, T^{+}=T^{-} t_{k} \in L_{k}, k=\overline{1, M}$, and on the shores of cracks, heat fluxes are given:

$\lambda_{k}^{*} \frac{\partial T^{ \pm}}{\partial n}=\tau_{k}\left(t_{k}\right) \pm \mu_{k}\left(t_{k}\right), t_{k} \in L_{k}, k=\overline{M+1, N}$.

In this case, the total amount of heat going through the contour $L=U L_{k}(k=\overline{M+1, N})$ is zero, that is,

$\sum_{k=M+1}^{N} \int_{L_{k}} \mu_{k}\left(t_{k}\right) \mathrm{ds}_{k}=0$.

Here $\mathrm{n}$ is the outer normal to the closed contour $L_{k}(k=$ $\overline{1, M})$ or to the left face of the cut $L_{k}(k=\overline{M+1, N}) ; \lambda\left(\lambda_{k}\right)$ is the coefficient of thermal conductivity of the matrix (inclusion $\left.S_{k}\right) ; T(x, y)$ is the temperature; $t_{k}$ is the complex coordinates of points on the contours $L_{k}$ in local coordinate systems; $s_{k}$ is the arc abscissa of the point; $\lambda_{k}^{*}=\lambda$ if the contour $L_{k}(k=$ $\overline{M+1, N}$ ) is located in the matrix and $\lambda_{k}^{*}=\lambda_{k}$ if it lies in the inclusion; the plus and minus indices indicate the boundary values of the corresponding values on the left and the right of the approach to the contour $L_{k}$.

In the problem of thermoelasticity, we assume that on the junction lines, the inclusions and the matrices $L_{n}(n=\overline{1, M})$ of the stress are continuous, and the displacement has a gap:

$\left[N_{n}\left(t_{n}\right)+\mathrm{i} T_{n}\left(t_{n}\right)\right]^{+}=\left[N_{n}\left(t_{n}\right)+\mathrm{i} T_{n}\left(t_{n}\right)\right]^{-}$,

$\left(u_{n}+\mathrm{i} v_{n}\right)^{+}-\left(u_{n}+\mathrm{i} v_{n}\right)^{-}=g_{n}^{*}\left(t_{n}\right), t_{n} \in L_{n}, n=\overline{1, M}$. (5)

Suppose that the plane is subjected to the action of a stationary temperature field $T(x, y)$. Assume that in the process of deformation, the crack lips are not in contact and they have a selfequilibrium load:

$\left[N_{n}\left(t_{n}\right)+\mathrm{i} T_{n}\left(t_{n}\right)\right]^{ \pm}=p_{n}\left(t_{n}\right), t_{n} \in L_{n}, n=\overline{M+1, N}$.

In relations (4), (5) and (6), $N_{n}\left(t_{n}\right)$ and $T_{n}\left(t_{n}\right)$ are the normal and tangential components of the load and $u_{n}, v_{n}$ are the components of displacement.

\section{THE PROBLEM SOLUTION}

\subsection{The system of integral equations of the problem of heat conduction}

We represent the temperature in the form $\mathrm{T}(\mathrm{x}, \mathrm{y})=$ $\operatorname{Ref}(\mathrm{z}), \mathrm{z} \in \mathrm{S}$ and use the complex temperature potential $\mathrm{F}(\mathrm{z})=\mathrm{f}^{\prime}(\mathrm{z})$ (Savruk,1981).

$$
\begin{aligned}
& F(z)=F_{0}(z)+\frac{1}{\mathrm{id}} \sum_{k=1}^{N} \int_{L_{k}} H_{k}\left(t_{k}\right) \operatorname{ctg}\left[\frac{\pi}{d}\left(T_{k}-z\right)\right] \mathrm{dt}_{k}, \\
& H_{k}\left(t_{k}\right)=\gamma_{k}^{\prime}\left(t_{k}\right)+i \mu_{k}\left(t_{k}\right) e^{-i \theta_{k}}, \gamma_{k}^{\prime}\left(t_{k}\right)=0(k=\overline{1, M}) .
\end{aligned}
$$

Here $f_{0}(z)=\int F_{0}(z) d z$ is the potential that determines the temperature field $T_{0}(x, y)$ in a continuous homogeneous plane without inclusions, and the function $T_{0}(x, y)$ is periodic with respect to the coordinate $x$ with period $d$.

By satisfying the boundary conditions (1) and (2) with the help of the complex temperature potential (7), we obtain in the heat conduction problem the system of N SIEs of the first and second kinds for the $N$ unknown functions $\mu_{k}\left(t_{k}\right)(k=\overline{1, M})$ on the contours of inclusions and $\gamma_{k}^{\prime}\left(t_{k}\right)(k=\overline{M+1, N})$ on the contours of cracks: 


$$
\begin{gathered}
i \delta_{n} H_{n}\left(t_{n}^{\prime}\right) e^{i \theta_{n}^{\prime}}-\frac{1}{d} \sum_{k=1}^{N} \int_{L_{k}} \operatorname{Im}\left[K_{\mathrm{nk}}\left(t_{k}, t_{n}^{\prime}\right) H_{k}\left(t_{k}\right) \mathrm{dt}_{k}\right]= \\
=\left(\delta_{n}-1\right) \tau\left(t_{n}^{\prime}\right) / \lambda_{n}^{*}+\Delta_{n} \operatorname{Im}\left[F_{0}\left(T_{n}^{\prime}\right) e^{i\left(\theta_{n}^{\prime}+\alpha_{n}\right)}\right], \\
t_{n}^{\prime} \in L_{n}, n=\overline{1, N} .
\end{gathered}
$$

Here $\delta_{n}=1$ for $n=\overline{1, M}$ and $\delta_{n}=0$ for $n=\overline{M+1, N}$, $T_{n}^{\prime}=t_{n}^{\prime} e^{i \alpha_{n}}+z_{n}^{0}$,

$K_{\mathrm{nk}}\left(t_{k}, t_{n}^{\prime}\right)=\frac{\Delta_{n}}{i} \operatorname{ctg}\left[\frac{\pi}{d}\left(T_{k}-T_{n}^{\prime}\right)\right] e^{i\left(\theta_{n}^{\prime}+\alpha_{n}\right)}$,

$\Delta_{n}=\left(\left(\lambda_{n}-\lambda\right) /\left(\lambda_{n}+\lambda\right)-1\right) \delta_{n}+1$.

The solution of system (8) must satisfy the conditions

$\int_{L_{n}} \gamma_{n}^{\prime}\left(t_{n}\right) \mathrm{dt}_{n}=0, n=\overline{M+1, N}$,

guaranteeing continuity of temperature in traversing the contours of cracks. Under condition (9), the system of integral equations (8) for its arbitrary right-hand side has a unique solution in the class of the functions (Savruk, 1981) $\gamma_{k}^{\prime}\left(t_{k}\right) \in H, t_{k} \in L_{k}(k=$ $\overline{1, M})$ and $\gamma_{k}^{\prime}\left(t_{k}\right) \in H^{*}, t_{k} \in L_{k}(k=\overline{M+1, N})$.

Note that the choice of the complex potential in form (7) ensures the identical satisfaction of the second equality in condition (1) based on such a choice of analytic continuation from the matrix region into the inclusion region, in which for the approach to the left and right of the contour of inclusion temperatures are equal.

\subsection{The system of integral equations of the problem of thermoelasticity}

Complex stress potentials can be represented in the form (Savruk,1981):

$$
\begin{aligned}
& \Phi(z)=\frac{1}{2 d} \sum_{k=1}^{N} \int_{L_{k}} Q_{k}\left(t_{k}\right) \operatorname{ctg}\left[\frac{\pi}{d}\left(T_{k}-z\right)\right] e^{i \alpha_{k}} \mathrm{~d} t_{k}, \\
& \Psi(z)=\frac{1}{2 d} \sum_{k=1}^{N} \int_{L_{k}} \overline{Q_{k}\left(t_{k}\right)} \operatorname{ctg}\left[\frac{\pi}{d}\left(T_{k}-z\right)\right] e^{-i \alpha_{k}} \overline{\mathrm{dt}_{k}}- \\
& -\left\{\begin{array}{c}
\operatorname{ctg}\left[\frac{\pi}{d}\left(T_{k}-z\right)\right]+ \\
-\frac{\pi}{d}\left(\overline{T_{k}}-T_{k}+z\right) \operatorname{cosec}^{2}\left[\frac{\pi}{d}\left(T_{k}-z\right)\right]
\end{array}\right\} Q_{k}\left(t_{k}\right) e^{i a_{k}} d t_{k},
\end{aligned}
$$

where:

$$
Q_{k}\left(t_{k}\right)=\left\{\begin{array}{l}
g_{k}\left(t_{k}\right), t_{k} \in L_{k}, k=\overline{1, M ;} \\
g_{k}^{\prime}\left(t_{k}\right)+\frac{i \beta_{*}}{1+\chi_{*}}\left[f^{+}\left(t_{k}\right)-f^{-}\left(t_{k}\right)\right], t_{k} \in L_{k},
\end{array}\right.
$$

$k=\overline{M+1, N}$.

We will assume that unknown functions $g_{k}^{\prime}\left(t_{k}\right)$ are sought in the class of the Hölder functions with integrable singularities at the tips of cracks: $g_{k}\left(t_{k}\right) \in H(k=\overline{1, M}), \quad g_{k}^{\prime}\left(t_{k}\right) \in$ $H^{*}(k=\overline{M+1, N})$. The functions $\mathrm{f}^{ \pm}\left(\mathrm{t}_{\mathrm{k}}\right)$ are the boundary values of the potential $\mathrm{f}(\mathrm{z})$.

By satisfying the boundary conditions (5) and (6) with the help of the complex potentials (10), we obtain in the problem of thermoelasticity, the system of $N$ SIEs of the first and second kinds for the $N$ unknown functions $Q_{k}\left(t_{k}\right)$ on the contours of inclusions $(\mathrm{k}=\overline{1, \mathrm{M}})$ and cracks $(\mathrm{k}=\overline{\mathrm{M}+1, \mathrm{~N}})$ :

$A_{n} Q_{n}\left(t_{n}^{\prime}\right)+\frac{1}{2 d} \sum_{k=1}^{N} \int_{L_{k}}\left[\begin{array}{l}K_{n k}\left(t_{k}, t_{n}^{\prime}\right) Q_{k}\left(t_{k}\right) d t_{k}+ \\ +L_{n k}\left(t_{k}, t_{n}^{\prime}\right) \overline{Q_{k}\left(t_{k}\right)} \overline{d t_{k}}\end{array}\right]$

$=R_{n}\left(t_{n}^{\prime}\right), t_{n}^{\prime} \in L_{n}, n=\overline{1, N}$,

where

$K_{\mathrm{nk}}\left(t_{k}, t_{n}^{\prime}\right)=e^{i \alpha_{k}}\left\{\begin{array}{c}B_{n} \operatorname{ctg}\left[\frac{\pi}{d}\left(T_{k}-T_{n}^{\prime}\right)\right]- \\ -D_{n} \frac{\overline{d t_{n}^{\prime}}}{d t_{n}^{\prime}} \operatorname{ctg}\left[\frac{\pi}{d}\left(T_{k}-T_{n}^{\prime}\right)\right] e^{-2 i \alpha_{n}}\end{array}\right\}$,

$L_{\mathrm{nk}}\left(t_{k}, t_{n}^{\prime}\right)$

$=-D_{n} e^{-i \alpha_{k}}\left\{\begin{array}{c}\left(1-\frac{\overline{d t_{n}^{\prime}}}{d t_{n}^{\prime}}\right) \operatorname{ctg}\left[\frac{\pi}{d}\left(T_{k}-T_{n}^{\prime}\right)\right] \\ -\frac{\pi}{d}\left(T_{k}-T_{n}^{\prime}+\overline{T_{n}^{\prime}}-\overline{T_{k}}\right) \\ \times \frac{\overline{d t_{n}^{\prime}}}{d t_{n}^{\prime}} \operatorname{cosec}^{2}\left[\frac{\pi}{d}\left(T_{k}-T_{n}^{\prime}\right)\right] \mathrm{e}^{-2 i \alpha_{\mathrm{n}}}\end{array}\right\}$,

$A_{n}=i \delta_{n}\left[1+\chi_{n}+\Gamma_{n}(1+\chi)\right] / 2, B_{n}=\left(\chi_{n}-\Gamma_{n} \chi-\right.$

1) $\delta_{n}+1, D_{n}=\left(2-\Gamma_{n}\right) \delta_{n}-1, \Gamma_{n}=G_{n} / G$,

$R_{n}\left(t_{n}^{\prime}\right)=\left[\Gamma_{n} \beta^{+} f^{-}\left(T_{n}^{\prime}\right)-\beta_{n}^{t} f^{+}\left(T_{n}^{\prime}\right)+2 G_{n} g_{n}^{\prime}\left(t_{n}^{\prime}\right)\right] \delta_{n}+$ $\left(1-\delta_{n}\right) p\left(t_{n}^{\prime}\right)$.

Here is the designation $\chi=(3-\mu) /(1+\mu)$, $\beta^{t}=\alpha^{t} E(1+\mu), \quad\left(\chi_{n}=\left(3-\mu_{n}\right) /\left(1+\mu_{n}\right), \beta_{n}^{t}=\alpha_{n}^{t}\right.$ $E_{n} /\left(1+\mu_{n}\right)$ for a plane stressed state; $\alpha^{t}, G, E\left(\alpha_{n}^{t}, G, E_{n}\right)$ is the coefficient of linear thermal expansion, shear modulus, Young's modulus; $\mu\left(\mu_{n}\right)$ is Poisson's ratio material of the matrix (respectively, inclusion).

In the class of the functions $g_{k}\left(t_{k}\right) \in H(k=\overline{1, M})$, $g_{k}^{\prime}\left(t_{k}\right) \in H^{*}(k=\overline{M+1, N})$, the system integral equation (11) has a unique solution for its arbitrary right-hand side under the conditions

$\int_{L n} g_{n}^{\prime}\left(t_{n}\right) \mathrm{dt}_{n}=0, n=\overline{M+1, N}$.

Condition (12) ensures the uniqueness of displacements in traversing the contours of cracks.

We note that the choice of complex potentials in equation (10) ensures the identical satisfaction of the boundary condition (4) on the contours of inclusions based on such a choice of an analytic continuation from the matrix region into the inclusion region, in which the stresses are continuous, and the displacements have a gap (for the approach to the contours of inclusions left and right). It should also be noted that condition (3) provides the limitation on the infinity of the function $f(z)$ and the stress component.

The SIFs $K_{I}$ and $K_{\mathrm{II}}$ at the crack tips are found according to formulas (Savruk,1981).

$K_{I}^{ \pm}-\mathrm{iK}_{\mathrm{II}}^{ \pm}=\mp \lim _{t_{k} \rightarrow l_{k}^{ \pm}}\left[\sqrt{2 \pi\left|t_{k}-l_{k}^{ \pm}\right|} Q_{k}\left(t_{k}\right)\right]$,

$k=\overline{M+1, N}$,

where the lower signs correspond to the beginning of the crack $\left(l_{k}^{-}\right)$and the upper ones correspond to the end of the crack $\left(l_{k}^{+}\right)$. 


\section{sciendo}

\section{THE NUMERICAL ANALYSIS}

Let in the band of periods of width $\mathrm{d}$ (along the axis $O x$ ), there is an elliptic inclusion with semi axes a and $b$, bounded by closed contour $L_{1}$ and an unloaded thermally insulated rectilinear crack $L_{2}\left(p_{2}\left(t_{2}\right)=\tau_{2}\left(t_{2}\right)=\mu_{2}\left(t_{2}\right)=0\right)$ with a length $2 l$. We will assume that along the closed contour $L_{1}$, the conditions for an ideal mechanical contact $\left(g_{1}^{*}\left(t_{1}\right)=0\right)$ are given,

The crack is located along the axis $O x\left(z_{2}^{0}=d_{1}, \alpha_{2}=0\right)$, and its center is at the point $\left(d_{1}, 0\right)$. At an infinitely distant point, a temperature field $T_{\infty}(x, y)=q(x \cos \gamma+y \sin \gamma)+T_{0}$ is given, where $T_{0}$ is the constant temperature and $q$ is the homogeneous heat flux at an angle $\gamma$ to the axis $O x$ (Fig. 2).
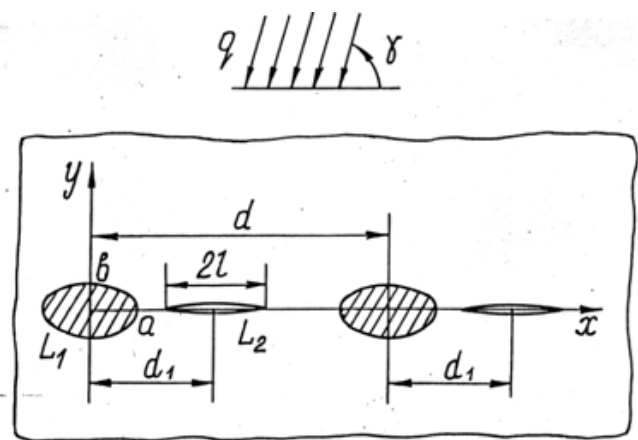

Fig. 2. A periodic system of elliptic inclusions and rectilinear cracks

The solution of the problem consists in solving the system of two integral equations (8) and (9) of the heat conduction problem with respect to functions $\mu\left(t_{1}\right), \gamma^{\prime}\left(t_{2}\right)$ and substituting them into the system of two equations (11) and (12) of the thermoelasticity problem for the determination of functions $Q_{k}\left(t_{k}\right), k=1,2$. Then we calculate the SIFs $K_{I}^{ \pm}, K_{I I}^{ \pm}$, which are the real quantities that characterise the stress-deformed state in the vicinity of the crack tips, according to formula (13). The numerical solution of the system of equations (8), (9), (12) and (13) is found using the method of mechanical quadrature (Erdogan et. el.; 1973). The dimensionless SIFs $K_{I}^{ \pm} / K_{0}, K_{I I}^{ \pm} / K_{0}$ where $K_{0}=q \beta^{t} l \sqrt{\pi l}$ $/(1+\chi)$ are obtained for different values of the thermal, mechanical and geometric parameters of the problem for $\chi=\chi_{1}=2$. In this case, if the heat flow $g$ is directed perpendicularly to the line of cracks $(\gamma=\pi / 2)$, then $K_{I}^{ \pm}=0$, $K_{I I}^{ \pm} \neq 0$; if parallelly $(\gamma=0)$, then $K_{I}^{ \pm} \neq 0, K_{I I}^{ \pm}=0$.

The numerical values in the numerator corresponding to $K_{\| l}^{-} / K_{0}$ (left tip of the crack), and in the denominator corresponding to $K_{l l}^{+} / K_{0}$ (right tip of the crack) are given in Table 1 . The values of SIFs in the left tip of the crack are greater (or lesser) than those in the right tip of the crack when $\lambda_{1} / \lambda<1$ (or $\lambda_{1} / \lambda>1$ ) for different ratio $\alpha_{1}^{t} / \alpha^{t}$.

We plotted the dependences of dimensionless SIFs $\mathrm{K}_{\mathrm{I}}^{ \pm} / \mathrm{K}_{0}$ on the half-length of the crack $\delta=1 / a$ for $\gamma=0, \frac{d_{1}}{a}=2$ and $\mathrm{d} / \mathrm{a}=6$ (Figs. 3 and 4). Curve 1 correspond to the value of $\Gamma_{1}=2$; curve 2 to that of $\Gamma_{1}=0.5$ for $\lambda_{1} / \lambda=0.5, \alpha_{1}^{t} / \alpha^{t}=$ 4 ; curves 3 to that of $\Gamma_{1}=2$; curves 4 to that of $\Gamma_{1}=0.5$ for $\lambda_{1} / \lambda=4, \alpha_{1}^{t} / \alpha^{t}=4$.
Tab. 1. The dimensionless numerical values $\mathrm{K}_{\|}^{ \pm} / \mathrm{K}_{0}$ for $\gamma=\pi / 2$, $\mathrm{b} / \mathrm{a}=0.5, \mathrm{l} / \mathrm{a}=0.8, \mathrm{~d}_{1} / \mathrm{a}=2$ depending on the relative values $\Gamma_{1}=G_{1} / G$ and different values of the parameters $\lambda_{1} / \lambda$,

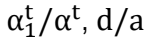

\begin{tabular}{|c|c|c|c|c|}
\hline \multirow{2}{*}{$\begin{array}{l}\Gamma_{1} \\
=\mathrm{G}_{1} / \mathrm{G}\end{array}$} & \multicolumn{2}{|c|}{$\lambda_{1} / \lambda=0.5$} & $\alpha_{1} / \alpha=4$ & \multicolumn{2}{c|}{$d / a=6$} \\
\cline { 2 - 5 } & $d / a=6$ & $d / a=12$ & $\begin{array}{c}\lambda_{1} / \lambda=4 \\
\alpha_{1}^{t} / \alpha^{t}=4\end{array}$ & $\begin{array}{c}\lambda_{1} / \lambda=0.5 \\
\alpha_{1}^{t} / \alpha^{t}=0.5\end{array}$ \\
\hline 0.2 & $-1.740 / 0.916$ & $-1.879 / 0.863$ & $-0.812 / 0.907$ & $-1.584 / 0.905$ \\
\hline 0.5 & $-1.754 / 1.006$ & $-2.181 / 0.984$ & $-0.710 / 0.969$ & $-1.401 / 0.974$ \\
\hline 2 & $-2.216 / 1.175$ & $-3.726 / 1.283$ & $-0.583 / 1.086$ & $-1.164 / 1.023$ \\
\hline 5 & $-2.921 / 1.348$ & $-5.795 / 1.601$ & $0.509 / 1.223$ & $-1.078 / 0.971$ \\
\hline 20 & $-3.844 / 2.044$ & $-9.556 / 2.388$ & $0.145 / 1.903$ & $-1.192 / 0.558$ \\
\hline
\end{tabular}

The solid lines correspond to the SIF $K_{\mathrm{I}}^{+} / K_{0}$ at the right crack tip and the dashed lines correspond to the SIF $K_{\mathrm{I}}^{-} / K_{0}$ at the left crack tip (closer to the inclusion). In this case, when the heat flux is directed in parallel $(\gamma=0)$ to the cracks and large axes of the elliptic inclusions $(b<a)$, the values of the SIFs at both tips of the crack are larger for the harder ones than the matrix $\left(G_{1}>G\right)$ inclusions compared with the case of less rigid $\left(G_{1}<G\right)$ inclusions when $\alpha_{1}^{t} / \alpha^{t}>1$ (Fig. 3). This means that the strength of the body increases with a decrease in the rigidity of inclusion in terms of fracture mechanics. If the large axes of the elliptic inclusions are perpendicular to the cracks and the heat flux $(b>a)$, then, on the contrary, the strength of the body decreases with a decrease in the stiffness of the inclusion (Fig. 4).

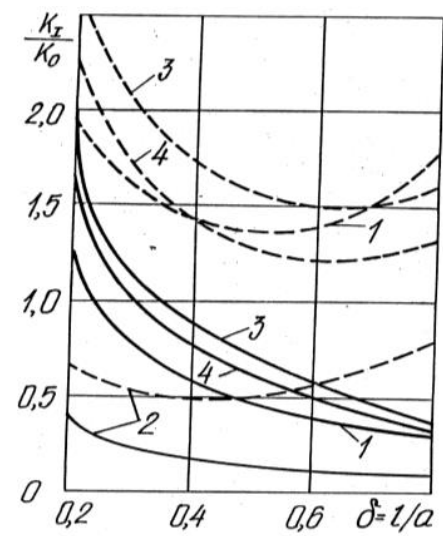

Fig. 3. Dependences of dimensionless SIFs $K_{I}^{ \pm} / K_{0}$ on the half-length of the crack $\delta=l / a$ for $b / a=0.5$

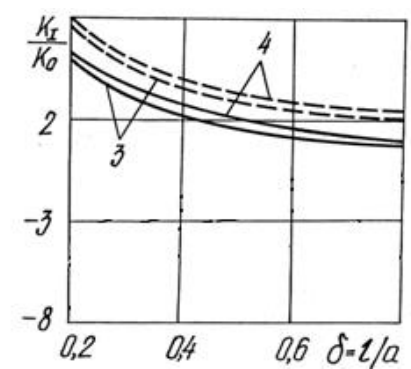

Fig. 4. Dependences of dimensionless SIFs $K_{I}^{ \pm} / K_{0}$ on the half-length of the crack $\delta=l / a$ for $b / a=2$

If the large axes of the inclusions are perpendicular to the direction of the heat flux and the cracks $(b>a)$, then the values of 
the SIF $K_{I}>0$ when $\lambda_{1} / \lambda>1$ (Fig. 4). If the large axes of inclusions are parallel $(b<a)$ to the direction of the heat flow, then the $K_{I}>0$ for different values of the ratio $\lambda_{1} / \lambda$ (Fig. 3).

It should also be noted that this does not take into account the possible contact of the shore of the crack. Therefore, in some cases, SIF $K_{I}<0$. Such a result can be used to obtain a solution of the problem using the method of superposition for the action, in addition to the given temperature field, other temperature or force factors, which together do not cause the contact of the shores of the crack.

In the considered problem, do not cause the contact of the shores of the crack if the heat flux is parallel to the large axes of the elliptic inclusions $(b<a)$ and the crack line and also when the flow is perpendicular $(b>a)$ for $\lambda_{1} / \lambda>1$. Then, according to the $\sigma_{\theta}$-criterion (based on the hypothesis of the initial growth of the crack) from equations of the boundary equilibrium (Panasyuk et al., 1976), it is possible to find the critical values of the heat flux $\mathrm{q}_{\mathrm{cr}}$ at which the growth of the crack begins and the local destruction of the body, according to the formula:

$$
q_{\text {cr }}=K_{q} \frac{K_{1 C}}{\cos ^{3} \frac{\theta_{*}}{2}\left(k_{1}^{ \pm}-3 k_{2}^{ \pm} \operatorname{tg} \frac{\theta_{*}}{2}\right)}, K_{q}=\frac{1+\chi}{\alpha^{t} \operatorname{El} \sqrt{\pi l}},
$$

where $K_{1 C}$ is the constant of a material that characterises the resistance of the material to the destruction and is determined experimentally; $\theta_{*}=2 \operatorname{arctg} \frac{k_{1}^{ \pm}-\sqrt{\left(k_{1}^{ \pm}\right)^{2}+8\left(k_{2}^{ \pm}\right)^{2}}}{4 k_{2}^{ \pm}}-$angle of initial growth of the crack, $k_{1}^{ \pm}=K_{I}^{ \pm} / K_{0}, k_{2}^{ \pm}=K_{I I}^{ \pm} / K_{0}$.

In the partial case, when the heat flux is directed parallelly to the cracks $(\gamma=0)$ for $\lambda_{1} / \lambda=4, \alpha_{1}^{t} / \alpha^{t}=4, \delta=l / a=0.6$, with $b / a=0.5$, obtained: if $\Gamma_{1}=2$, then $q_{\mathrm{cr}}=0.86 K_{q} K_{1 C}$; if $\Gamma_{1}=0,5$, then $q_{\mathrm{cr}}=1.04 K_{q} K_{1 c}$; when $b / a=2$, received: if $\Gamma_{1}=2$, then $q_{\mathrm{cr}}=0.54 K_{q} K_{1 C}$; if $\Gamma_{1}=0.5$, then $q_{\mathrm{cr}}=$ $0.44 K_{q} K_{1 C}$.

On the basis of the analysis of the obtained critical values of the heat flow, it follows that a more rigid than an elliptic inclusion matrix with a large axis parallel to the heat flux $(b<a)$ determines a less critical value of the heat flux $q_{\mathrm{cr}}$ (at which the growth of the crack in the left tip begins) when compared with less tough inclusion. If the large axis of elliptic inclusions is perpendicular to the heat flux $(b>a)$, then, on the contrary, it causes an increase. A similar situation is observed for the right tip of the crack.

\section{CONCLUSIONS}

Two-dimensional mathematical models of the problems of stationary heat conductivity and thermoelasticity for an infinite elastic plane with a periodic system of curvilinear inclusions and cracks in the form of system SIEs of the first kind on the contours of cracks and the second kind on the contours of inclusions are constructed. This approach allows to obtain a numerical solution of system SIE by applying the method of mechanical quadrature .

A numerical solution of system SIE in the partial case of a plane with a periodic system of elliptic inclusions and rectilinear thermally insulated cracks in the action of a given heat flux is obtained. On the basis of this solution, SIF at the crack tips are calculated, which in the future are used to determine the critical values of the heat flux for which the crack begins to grow.

\section{REFERENCES}

1. Brock L.M. (2016), Contours for planar cracks growing in three dimensions: Coupled thermoelastic solid (planar crack growth in 3D), Journal of Thermal Stresses, 39(3), 345-359.

2. Cheesman B.A., Santare M.H. (2000), The interaction of a curved crack with a circular elastic inclusion, Int. J. Fract.,103, 259-278.

3. Chen H., Wang Q., Liu G. , Sun J. (2016), Simulation of thermoelastic crack problems using singular edge-based smoothed finite element method, Int. J. of Mech. Sci., 115,116, 23-134.

4. Choi H.J. (2014), Thermoelastic interaction of two offset interfacial cracks in bonded dissimilar half-planes with a functionally graded interlayer, Acta Mechanica , 225(7),2111-2131.

5. Elfakhakhre N.R.F., Nik L., Eshkuvatov N.M.A. (2017), Stress intensity factor for multiple cracks in half plane elasticity, AIP Conference, 1795(1).

6. Erdogan F., Gupta B.D., Cook T.S. (1973), The numerical solutions of singular integral equations, Methods of analysis and solutions of crack problems. Leyden: Noordhoff Intern. publ., 368-425.

7. Havrysh V.I. (2015), Nonlinear boundary-value problem of heat conduction for a layered plate with inclusion, Phis.-chim. mechanica materialiv (Materials Science), 51(3), 331-339.

8. Havrysh V.I. (2017) Investigation of temperature fields in a heatsensitive layer with through inclusion, Phis.-chim.mechanica materialiv (Materials Science), 52(4), 514-521.

9. Kit H.S., Chernyak M.S. (2010), Stressed state of bodies with thermal cylindrical inclusions an cracks (plane deformation), Phis.-chim. mechanica materialiv (Materials Science), 46(3), 315-324.

10. Kit, G.S. , Ivas'ko, N.M. (2013), Plane deformation of a semi-infinite body with a heat-active crack perpendicular to its boundary, Teoret. I prikl. Mehanika, 53(7), 30-37.

11. Matysiak S.J., Evtushenko A.A., Zelenjak V.M. (1999), Frictional heating of a half-space with cracks. I. Single or periodic system of subsurface cracks, Tribology Int., 32, 237-243.

12. Panasyuk V.V., Savruk M.P., Datsyshyn O.P. (1976), Distribution tense neighborhood of cracks in the plates and shells (in Russian), Kiev, Naukova dumka

13. Rashidova E.V., Sobol B.V. (2017), An equilibrium internal transverse crack in a composite elastic half-plane, Journal of Applied Mathmatics and Mechanics, 81(3), 236-247

14. Savruk M.P., Zelenyak V.M. (1986), Singular integral equations of plane problems of thermal conductivity and thermoelasticity for a piecewise-uniform plane with cracks, Phis.-chim. mechanica materialiv (Materials Science), 22( 3), 297-307.

15. Savruk M.P. (1981), Two-dimensional elasticity problem for bodies with cracks (in Russian), Kiev, Naukova dumka.

16. Sekine H. (1975), Thermal stress singularities at tips of a crack in a semi-infinite medium under uniform heat flow, Eng. Fract. Mech. , 7 (4) , 713-729.

17. Sushko O.P. (2013), Thermoelastic state of a body with two coplanar thermally active circular cracks, Journal of Mathematical Sciences, 190(5), 725-739.

18. Tweed I., Lowe S. (1979), The thermoelastic problem for a halfplane with an internal line crack, Int. J. Eng. Sci., 17(4), 357-363.

19. Xiao Z..M., Chen B.J. (2001), Stress intensity factor for a Griffith crack interacting with a coated inclusion, Int. J. Fract., 108, 193-205.

20. Zelenyak B.M. (2015), Thermoelastic equilibrium of a three-layer circular hollow cylinder weakened by a crack, Phis.-chim. mechanica materialiv (Materials Science), 50(1), 14-19.

21. Zelenyak V.M. (2012), Thermoelastic interaction of a twocomponent circular inclusion with a crack in the plate, Phis.-chim. mechanica materialiv (Materials Science), Materials Science, 48( 3), 301-307.

22. Zelenyak, V.M., Kolyasa, L.I. ( 2016). Thermoelastic state of a half plane with curvilinear crack under the conditions of local heating, Phis.-chim. mechanica materialiv (Materials Science), 52(3), 315-322 